CARDIOVASCULAR MEDICINE

\title{
Cardiac magnetic resonance imaging: long term reproducibility of the late enhancement signal in patients with chronic coronary artery disease
}

\author{
H Bülow, C Klein, I Kuehn, R Hollweck, S G Nekolla, K Schreiber, F Haas, J Böhm, \\ B Schnackenburg, R Lange, M Schwaiger
}

Heart 2005;91:1158-1163. doi: 10.1136/hrt.2004.045609

See end of article for authors' affiliations

.....................

Correspondence to: Dr Hubertus Bülow, Nuklearmedizinische Klinik und Poliklinik, Technische Universitä München, Klinikum rechts der Isar, IsmaningerStrasse 22, 81675 Munich, Germany; h. buelow@|rz.tu-muenchen. de

Accepted 29 October 2004

\begin{abstract}
Objective: To determine long term reproducibility of the late enhancement (LE) signal in contrast enhanced magnetic resonance imaging (MRI) and potential changes of the signal after revascularisation. Methods: 33 patients (29 men, mean (SD) 61 (11) years) with coronary artery disease (CAD) and left ventricular dysfunction (ejection fraction 30 (7)\%) underwent two contrast enhanced MRI procedures within 9 (3) months. Fiffeen patients (group A: 14 men, 59 (12) years) had no interventions between the two studies. Eighteen patients underwent revascularisation after MRI 1 (group B: 15 men, 62 (9) years). Changes in the LE signal between the first and second MRIs were investigated in both groups as well as intraobserver and interobserver variabilities for delineation of the signal.

Results: The LE signal was highly reproducible in groups A and B for segmental analysis (concordance $86 \% \vee 82 \%$, respectively; $\kappa=0.70 v 0.67$ ) and summed scores (group $A: r=0.97, p<0.001$; group $B$ : $r=0.93, p<0.001)$. The LE signal was quantified as $27(27) \mathrm{cm}^{3}$ in group $A$ versus $30(16) \mathrm{cm}^{3}$ in group $B$ in the first MRI and $26(25) \mathrm{cm}^{3}$ versus $30(15) \mathrm{cm}^{3}$, respectively, for the second MRI (both not significant). Moreover, low intraobserver and interobserver variabilities were observed in segmental analysis ( $\kappa=0.86$ and 0.74 , respectively, for group $A$, and $\kappa=0.87$ and 0.82 , respectively, for group $B$ ). Conclusion: In patients with chronic CAD, the LE signal in contrast enhanced MRI is very stable over an extended time period. These results further characterise contrast enhanced MRI as a useful tool for myocardial viability assessment. Low intraobserver and interobserver variabilities promise robustness of the method for clinical application.
\end{abstract}

t has been shown in several animal studies that the area of the late enhancement (LE) signal in contrast enhanced magnetic resonance imaging (MRI) after intravenous injection of gadolinium based contrast agents correlates well with location and extent of histologically proven myocyte necrosis after acute ${ }^{1-4}$ and chronic ${ }^{34}$ myocardial infarction. The exact mechanism of delayed contrast enhancement in myocardial infarction is not yet understood. However, delayed wash-in and wash-out of the contrast agent and an increased distribution volume may have a role. ${ }^{25}$

In clinical settings, delineation of non-viable myocardium by the presence of an LE signal was validated by either decreased tracer uptake in nuclear studies $^{5-9}$ or loss of contractile reserve shown by echocardiography.

Studies investigating the time course of the LE signal after acute and subacute myocardial infarction observed a decrease in size of the signal by a factor of 2 to $3.5 .^{3}{ }^{4}$ A recent study evaluating short term variability reported excellent reproducibility of the LE signal in two MRIs recorded within a few minutes in patients with chronic coronary artery disease (CAD). ${ }^{10}$ In contrast, no information exists about long term reproducibility of the LE signal in patients with stable CAD and absence of recent cardiac events. Before revascularisation, several clinical studies showed the usefulness of the LE signal as a predictor of functional improvement in impaired myocardium. ${ }^{11-14}$ There are no data, however, concerning potential reversibility of the LE signal, which may be caused by reversing left ventricular (LV) dysfunction (hibernating myocardium)

We therefore investigated long term reproducibility of the LE signal in patients with CAD with impaired LV function and absence of cardiac events or interventions, as well as in a patient population with revascularisation, to further characterise contrast enhanced MRI as a tool for myocardial viability assessment.

\section{METHODS}

\section{Patients}

Fifty four patients with angiographically proven CAD and impaired LV function were initially enrolled in the study. Of the 54 patients, 15 declined a follow up MRI, three patients underwent implantation of a cardioverter-defibrillator, and three patients died before the follow up. The remaining 33 patients (29 men, mean (SD) age 60.7 (10.6) years) were studied twice with MRI within an interval of 9 (3) months (range 3-15 months). Four of the 33 patients had single vessel disease, three had double vessel disease, and 26 had triple vessel disease. Mean (SD) LV ejection fraction (LVEF) was $30(7) \%)$. Twenty one patients had a history of prior myocardial infarction and 22 patients had undergone revascularisation procedures (bypass graft surgery, 11 patients; percutaneous coronary intervention, 13 patients), neither of which had occurred within two months before the first MRI. In the time interval between both scans, 15 patients (group A: 14 men, mean (SD) age 59.3 (12.3) years) were treated medically only, with no patients undergoing any coronary intervention or having a cardiac event before the

Abbreviations: CAD, coronary artery disease; DTPA, diethylenetriaminepentaacetic acid; LE, late enhancement; LV, left ventricular; LVEF, left ventricular ejection fraction; MRI, magnetic resonance imaging; WM, wall motion 
second MRI. The other 18 patients (group B: 15 men, mean (SD) age 61.8 (9.2) years) were revascularised within five weeks after the first MRI. Fifteen patients underwent bypass graft surgery ( six patients had two bypass grafts, five patients had three bypass grafts, three patients had four bypass grafts, and one patient had one bypass graft) and three patients had a percutaneous coronary intervention (one native vessel/ bypass graft each). After revascularisation, one patient had a coronary reintervention (percutaneous transluminal coronary angioplasty of one native vessel) three months before the second MRI. For all patients of group B, the time interval between revascularisation and the second MRI was at least three months. In between the scans, no patient of this group had a cardiac event. Written informed consent was obtained from all patients after approval by the local ethics committee.

\section{Magnetic resonance imaging}

MRIs were recorded with two 1.5 T scanners (Gyroscan ACS 1.5T, Philips Medical Systems, Best, the Netherlands (22 patients) and Magnetom Sonata Maestro Class, Siemens Medical Solutions, Erlangen, Germany (11 patients)) with a dedicated cardiac phased array surface coil. For comparison, both scans for each patient were recorded with the same scanner and by the same physician $\left(\begin{array}{ll}H & B\end{array}\right)$. Ten to 13 consecutive cine short axis views and one, two, and four chamber view, each acquired during one breath hold, were recorded for evaluation of wall motion (WM) and LVEF in a steady state free precession technique (Gyroscan ACS: echo time $1.4 \mathrm{~ms}$, repetition time $2.9 \mathrm{~ms}$, slice thickness $8 \mathrm{~mm}$, spatial resolution $1.4 \times 1.2 \mathrm{~mm}^{2}$, flip angle $60^{\circ}$, temporal resolution $42 \mathrm{~ms}$; Magnetom Sonata: echo time $1.5 \mathrm{~ms}$, repetition time $2.6 \mathrm{~ms}$, slice thickness $8 \mathrm{~mm}$, spatial resolution $2.2 \times 1.3 \mathrm{~mm}^{2}$, flip angle $65^{\circ}$, temporal resolution $35 \mathrm{~ms})$. The LE signal was evaluated by two techniques 1520 minutes after intravenous injection of Gd-diethylenetriaminepentaacetic acid (DTPA) $0.2 \mathrm{mmol} / \mathrm{kg}$ body weight (Magnevist; Schering AG, Berlin, Germany): an inversion recovery three dimensional turbo gradient echo technique with echo planar readout (Gyroscan ACS: echo time $3.3 \mathrm{ms,}$ repetition time $5.4 \mathrm{~ms}$, echo planar imaging factor 11 , slice thickness $5 \mathrm{~mm}$, spatial resolution $1.2-1.2 \mathrm{~mm}^{2}$, flip angle $15^{\circ}$, acquisition window per heart beat $284 \mathrm{~ms}$, inversion time varied visually to achieve complete nulling of normal myocardium between 225-300 ms for MRI l and kept constant for MRI 2); or a Tl weighted two dimensional inversion recovery true fast imaging technique with steady state precession sequence (Magnetom Sonata: echo time $1.4 \mathrm{~ms}$, repetition time $2.4 \mathrm{~ms}$, slice thickness $8 \mathrm{~mm}$, spatial resolution $2.8 \times 1.4 \mathrm{~mm}^{2}$, flip angle $60^{\circ}$, acquisition window $156 \mathrm{~ms} /$ heart beat, inversion time varied between 225$300 \mathrm{~ms}$ for MRI 1 and kept constant for MRI 2). Both inversion recovery sequences were acquired at end diastole, covering the whole LV (in short axis views and part of the LV in two and four chamber views).

\section{Data analysis}

MRIs were evaluated with either a commercially available software (Mass 4.0; Medis, Leiden, the Netherlands for WM and LE data; Gyroscan ACS, Argus, Siemens Medical Solutions for WM data; Magnetom Sonata) or an in-house developed software (Munich Heart for LE data; Magnetom Sonata). Analysis of cine short axis views yielded LVEF and information about regional WM. For visual evaluation of WM and LE, three representative and corresponding short axis views (apical, mid-ventricular, and basal) and the apex of the LV were divided into 33 segments as recently described. ${ }^{9}$ Quality of WM was scored by a four point system (0, normal WM; 1, mild hypokinesis; 2, severe hypokinesis; 3, akinesis/ dyskinesis) and extent of LE was scored by a three point
Table 1 Segmental analysis of the late enhancement (LE) signal for groups A and B

\begin{tabular}{|c|c|c|c|c|}
\hline & \multicolumn{2}{|c|}{$\begin{array}{l}\text { Group A } \\
\text { ( } n=495 \text { segments) }\end{array}$} & \multicolumn{2}{|c|}{$\begin{array}{l}\text { Group B } \\
\text { ( } \mathrm{n}=594 \text { segments) }\end{array}$} \\
\hline & MRI 1 & MRI 2 & MRI 1 & MRI 2 \\
\hline \multicolumn{5}{|l|}{ LE score } \\
\hline 0 & 343 (70\%) & $338(68 \%)$ & $350(59 \%)$ & $358(60 \%)$ \\
\hline 1 & $76(15 \%)$ & $68(14 \%)$ & $174(29 \%)$ & $168(28 \%)$ \\
\hline 2 & $76(15 \%)$ & $89(18 \%)$ & $70(12 \%)$ & $68(12 \%)$ \\
\hline Concordance & $424(86 \%)$ & & $486(82 \%)$ & \\
\hline$\kappa$ & 0.70 & & 0.67 & \\
\hline$P(\kappa)$ & $<0.001$ & & $<0.001$ & \\
\hline
\end{tabular}

system (0, none; 1, non-transmural, defined arbitrarily as containing less than $50 \%$ of a transmural signal; and 2 , transmural enhancement, defined arbitrarily as containing at least $50 \%$ of a transmural signal). All myocardial segments were evaluated according to this grading system and a summed score of LE and WM was calculated for each patient. The size of the LE signal (in cubic centimetres) was quantified from the regions of interest including the area of the LE signal on each short axis slice covering the LV.

Two independent observers determined interobserver variability of visual LE signal analysis by reading MRI 1 of the first 22 patients and were blinded to each other and to clinical data. To analyse intraobserver variability of the contrast enhanced MRI, one observer evaluated MRI l of the first 22 patients at two times at an interval of three months and was blinded to clinical data and results of the first evaluation.

\section{Statistical analysis}

Cardinal and ordinal variables were expressed as mean (SD) or range, where appropriate. Discrete variables are shown as absolute and relative frequencies. For evaluation of differences between groups $\mathrm{A}$ and $\mathrm{B}$ and between the two dimensional and three dimensional imaging sequences Mann-Whitney U test was applied, and for changes between MRI 1 and MRI 2 the Wilcoxon test was performed. Summed scores and the amount of LE signal were compared by Spearman correlation coefficients. Bland-Altman plots were created to evaluate agreement in the LE score and quantification of the LE signal between MRI 1 and MRI 2. Time course and interobserver and intraobserver agreements of the LE signal on a segmental basis were assessed by $\kappa$ statistics. Bonferroni adjustment was used to account for multiple testing. Computed $p$ values were two sided and $p<0.05$ was

Table 2 Segmental analysis of the LE signal showing concordance between the first and second MRIs for groups $A$ and $B$

\begin{tabular}{lllll}
\hline & \multicolumn{2}{l}{ LE score (MRI 2) } & \\
\cline { 2 - 4 } LE score (MRI 1) & $\mathbf{0}$ & $\mathbf{1}$ & $\mathbf{2}$ & Total \\
\hline Group A & 323 & 17 & 3 & 343 \\
0 & 11 & 40 & 25 & 76 \\
1 & 4 & 11 & 61 & 76 \\
2 & 338 & 68 & 89 & 495 \\
Total & 314 & 30 & 6 & 350 \\
Group B & 40 & 122 & 12 & 174 \\
0 & 4 & 16 & 50 & 70 \\
1 & 358 & 168 & 68 & 594 \\
2 & & & & \\
Total & & &
\end{tabular}




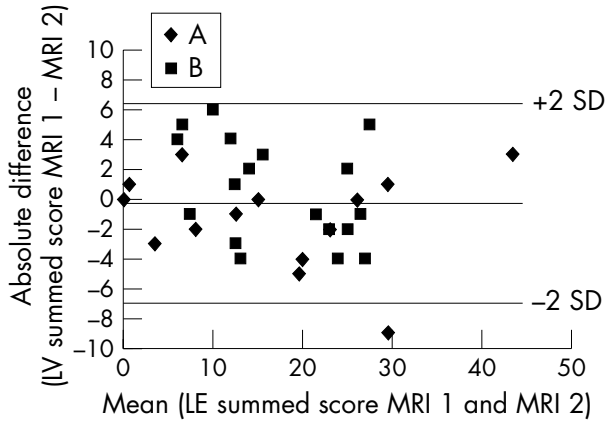

Figure 1 Bland-Altman plot comparing late enhancement (LE) summed scores (patient groups A and B) between the first (MRI 1) and second magnetic resonance images (MRI 2).

considered significant. Data were analysed with SPSS 11.5 (SPSS Inc, Chicago, Illinois, USA).

\section{RESULTS}

\section{Segmental LE signal analysis}

Tables 1 and 2 list the results of segmental LE signal analysis for both MRIs in patient groups A and B. Both scans were highly concordant in group A (concordance in $86 \%$ (424 of 495 ) of segments) but also in group B (82\% (486 of 594)). Kappa statistics indicated close agreement (group A: $\kappa=0.70$; group $\mathrm{B}: \kappa=0.67)$, being highly significant $(p<0.001$ each $)$. Analysis of a subgroup of group B of only patients with improved LV function (15 patients) showed concordance in $82 \%$ (407 of 495 ) of segments and $\kappa=0.68$, therefore not different from group A and group B as a whole. Analysis of the LE summed scores for each patient showed a close correlation between MRI 1 and MRI 2 for both patient groups (group A: $r=0.99, \mathrm{p}<0.001$; group B: $r=0.98$, $\mathrm{p}<0.001)$ and the Bland-Altman plot showed good agreement (fig 1). The two imaging sequences did not differ significantly according to the LE summed scores (group A: $\mathrm{p}=0.87$; group $\mathrm{B}: \mathrm{p}=0.86)$. Figures 2 and 3 show examples from three patients of group A and group B, respectively.

\section{Intraobserver and interobserver variability in LE signal evaluation}

Analysis of intraobserver and interobserver variabilities in the segmental evaluation of MRIs from the first 22 patients (group A: 13 patients; group B: nine patients.) showed agreement in $93 \%$ (398 of 426 segments) and $88 \%$ (375 of 426 ), respectively, for group A and $92 \%$ (274 of 297) and $89 \%$ (265 of 297), respectively for group B. The corresponding $\kappa$ values were $0.86(\mathrm{p}<0.001)$ and $0.74(\mathrm{p}<0.001)$, respectively, for group A and $0.87(\mathrm{p}<0.001)$ and 0.82 $(\mathrm{p}<0.001)$, respectively, for group B. All agreements were highly significant with no significant differences between group $\mathrm{A}$ and $\mathrm{B}$.

\section{Quantification of the LE signal}

The mean (SD) LE signal area in MRI 1 was quantitatively assessed to be $27(27) \mathrm{cm}^{3}$ in group A and $30(16) \mathrm{cm}^{3}$ in group B. The corresponding values for MRI 2 were 26 (25) $\mathrm{cm}^{3}$ and $30(15) \mathrm{cm}^{3}$, respectively. For both patient groups, no significant changes were observed between the first and second scans (group A: MRI $1 v 2, \mathrm{p}=0.311$; group $\mathrm{B}: \mathrm{p}=0.879$ ) or between the two groups at each scan (MRI 1: A $v \mathrm{~B}, \mathrm{p}=0.69$; MRI 2: $\mathrm{p}=0.62$ ). The quantitative amount of LE signal in MRI 1 and MRI 2 correlated closely in both patient groups (group A: $r=0.99, \mathrm{p}<0.001$; group B: $r=0.98, \mathrm{p}<0.001)$. Furthermore, the Bland-Altman plot indicated good agreement between the absolute amount of LE signal in both scans (fig 4), with the majority of values falling within $\pm 2 \mathrm{SD}$ of the mean difference. A comparison between both imaging sequences (two versus three dimensional) showed no significant differences (group A: $\mathrm{p}=0.22$; group $\mathrm{B}: \mathrm{p}=0.50$. The quantitative LE signals of both sequences in both MRIs were well correlated.

\section{Analysis of LE signal in relation to LV function}

Between the baseline and follow up scans the LVEF, indicating global LV function, did not change significantly in group A (31 (8)\% $v 32(8) \%$ in the first and second MRIs, respectively, $\mathrm{p}=0.20)$ but improved non-significantly in group B (28 (6)\% $v 32(8) \%, p=0.052)$. For both MRIs, no significant differences in LVEF were found between patient groups $\mathrm{A}$ and $\mathrm{B}$.

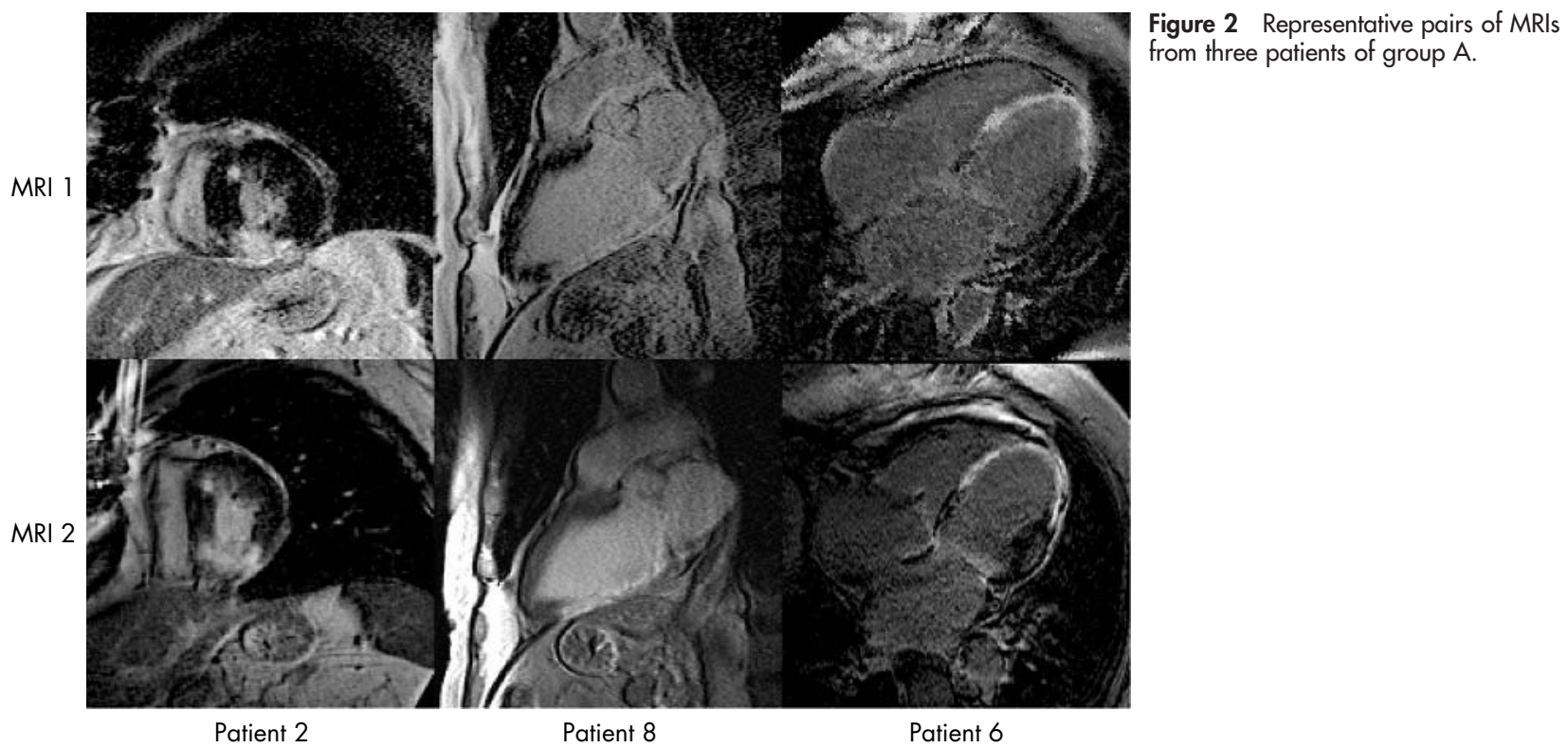




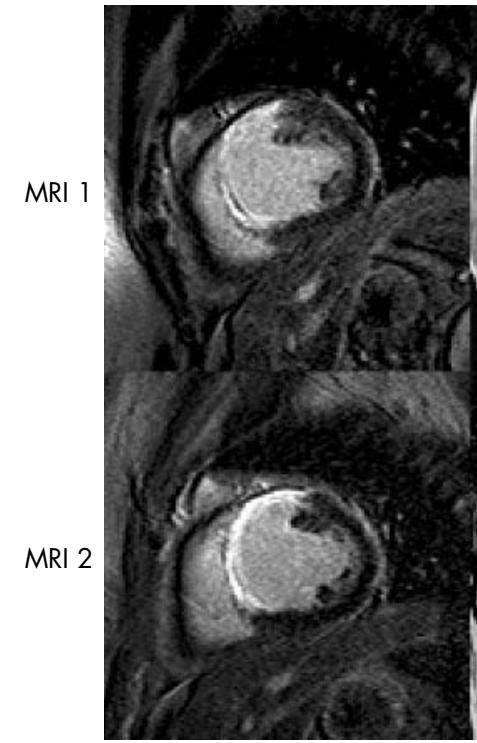

Patient 18

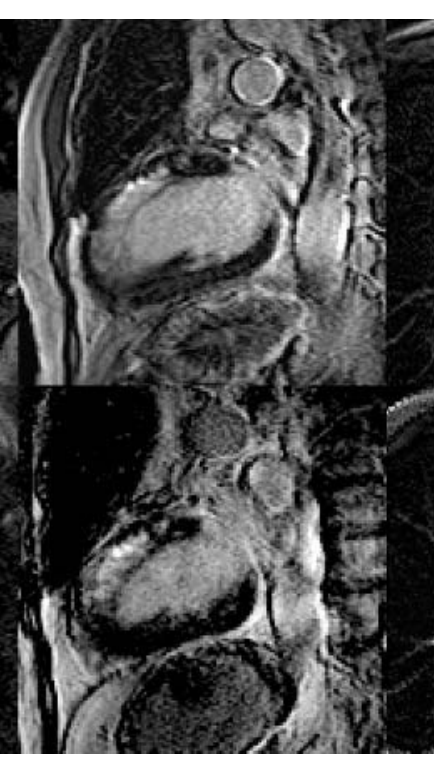

Patient 21

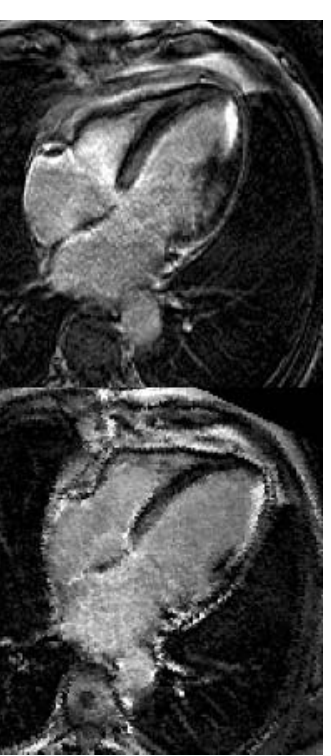

Patient 23
Figure 3 Representative pairs of MRIs from three patients of group $B$.
Table 3 shows the relation between LE score and WM score on a segmental basis. Either severe hypokinesis or akinesis/ dyskinesis (WM score 2 or 3) was observed in $88 \%$ of segments containing transmural contrast enhancement (LE score 2) but in only $36 \%$ of segments with no LE signal. Table 4 lists the mean segmental LE score for each degree of WM score in both MRIs and both patient groups. A significant correlation between the WM score and the mean segmental LE score was found in both patient groups and both MRIs (group A, MRI 1 and 2: $r=0.51$ and $r=0.52$, respectively, $\mathrm{p}<0.001$ each; group B, MRI 1 and 2: $r=0.55$ and $r=0.51, \mathrm{p}<0.001$ each).

Table 5 shows the observed changes of WM score between MRI 1 and 2 according to LE score. With increasing LE score the percentage of segments with no change in WM score increased (LE score 0: 62\%; LE score 1: 74\%; and LE score 2: $90 \%)$. Conversely, the lower the LE score, the higher the percentage of segments with improving WM score, especially in group B (LE score 0: 19\% v 27\%; LE score 1: $18 \% v 18 \%$; and LE score 2: $7 \% v 3 \%$ in group A $v$ group B, respectively). Of 213 dysfunctional segments containing no LE signal (LE score 0) at MRI 1, 94 (44\%) improved functionally after revascularisation. Segments with transmural enhancement (LE score 2) had a worsening (5\% of segments) or improved WM score $(5 \%)$. Seventy one per cent of these had only minor changes at the dysfunctional edge of the WM scale (changes between WM score 2 and 3). The mean summed WM score

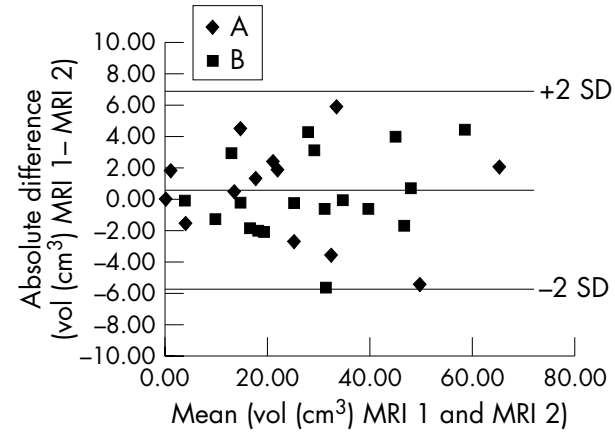

Figure 4 Bland-Altman plot comparing quantitative assessment of the LE signal (volume (vol) in $\mathrm{cm}^{3}$ ) in patient groups $A$ and $B$ in both MRIs. for patient group A did not change significantly between the two scans ( $51 v 5 \mathrm{l}, \mathrm{p}=0.40$ ), whereas it tended to improve, though not significantly, in group B $(46 v 50, \mathrm{p}=0.15$.

\section{DISCUSSION}

The results of this study show that, in patients with chronic CAD and impaired LV function, the extent and location of the LE signal are highly reproducible over an extended time. Furthermore, the LE signal was stable regardless of whether patients had undergone conservative treatment or revascularisation.

Analysis of the LE signal, together with regional WM, showed increasing regional dysfunction with increasing

Table 3 Segmental LE and wall motion (WM) scores in MRI 1 for groups A and B

\begin{tabular}{|c|c|c|c|c|c|}
\hline \multirow[b]{2}{*}{ LE score } & \multicolumn{4}{|c|}{ WM score } & \multirow[b]{2}{*}{ Tota } \\
\hline & 0 & 1 & 2 & 3 & \\
\hline \multicolumn{6}{|l|}{ Group A } \\
\hline 0 & 119 & 90 & 91 & 43 & 343 \\
\hline 1 & 5 & 10 & 25 & 36 & 76 \\
\hline 2 & 2 & 7 & 11 & 56 & 76 \\
\hline Total & 126 & 107 & 127 & 135 & 495 \\
\hline \multicolumn{6}{|l|}{ Group B } \\
\hline 0 & 137 & 98 & 83 & 32 & 350 \\
\hline 1 & 9 & 26 & 75 & 64 & 174 \\
\hline 2 & 1 & 7 & 15 & 47 & 70 \\
\hline Total & 147 & 131 & 173 & 143 & 594 \\
\hline
\end{tabular}

Table 4 Mean (SD) segmental LE score for each degree of WM score in both MRIs and both patient groups

\begin{tabular}{llllll}
\hline \multirow{2}{*}{$\begin{array}{l}\text { WM } \\
\text { score }\end{array}$} & \multicolumn{2}{l}{ MRI 1 mean LE score } & & \multicolumn{2}{l}{ MRI 2 mean LE score } \\
\cline { 2 - 3 } \cline { 5 - 6 } & Group A & Group B & & Group A & Group B \\
\hline 0 & $0.07(0.31)$ & $0.07(0.29)$ & & $0.08(0.30)$ & $0.15(0.41)$ \\
1 & $0.22(0.55)$ & $0.31(0.57)$ & & $0.23(0.55)$ & $0.33(0.54)$ \\
2 & $0.37(0.64)$ & $0.61(0.64)$ & & $0.38(0.67)$ & $0.57(0.64)$ \\
3 & $1.10(0.85)$ & $1.10(0.74)$ & & $1.23(0.87)$ & $1.14(0.87)$ \\
\hline
\end{tabular}




\begin{tabular}{|c|c|c|c|c|}
\hline \multirow[b]{2}{*}{ LE score } & \multicolumn{3}{|l|}{$\Delta \mathrm{WM}$ score } & \multirow[b]{2}{*}{ Total } \\
\hline & No change & Worsened & Improved & \\
\hline \multicolumn{5}{|l|}{ Group A } \\
\hline 0 & 221 & 57 & 65 & 343 \\
\hline 1 & 54 & 8 & 14 & 76 \\
\hline 2 & 67 & 4 & 5 & 76 \\
\hline Total & 342 & 69 & 84 & 495 \\
\hline \multicolumn{5}{|l|}{ Group B } \\
\hline 0 & 208 & 48 & 94 & 350 \\
\hline 1 & 132 & 10 & 32 & 174 \\
\hline 2 & 65 & 3 & 2 & 70 \\
\hline Total & 405 & 61 & 128 & 594 \\
\hline
\end{tabular}

transmurality of contrast enhancement. In addition, improvement in WM decreased with increasing transmurality of the LE signal. This is in concordance with results of several studies describing a relation between the degree of myocardial contrast enhancement and the probability of functional recovery after revascularisation. ${ }^{11}{ }^{13} 14$

Concerning possible temporal changes of the LE signal, there are only few data published. One recent study by Mahrholdt and colleagues ${ }^{10}$ evaluated short term reproducibility of contrast enhanced MRI in 20 patients with chronic myocardial infarction. They found high reproducibility of the LE signal 10 and 25 minutes after one injection of a gadolinium based contrast agent and repositioning of the patients between the two scans. In contrast, patients in our study underwent two MRIs with a mean time interval of 9 (3) months, therefore addressing biological and to a lesser degree methodological reproducibility. Previous studies investigating LE signal changes at different time points focused mainly on the time course after acute and subacute myocardial infarction. In a canine model, Kim and colleagues $^{3}$ studied seven dogs three days and eight weeks after acute infarction. They found a decrease in the extent of hyperenhancement by a factor greater than 3, which was explained by shrinkage of scar tissue. This is in accordance with a histological study in a canine model by Reimer and colleagues, ${ }^{15}$ who described a decrease of infarct size in the subacute phase by a factor of 4 . In a porcine study, however, van Dijkman and colleagues ${ }^{16}$ found little change in contrast enhancement between one and three weeks after coronary occlusion. According to their medical records, none of our patients had an acute myocardial infarction within two months before the first or in between the two MRIs. Thus, no significant changes in infarct size based on shrinkage of infarcted myocardium could be expected, as the results confirmed.

In patients undergoing bypass graft surgery or percutaneous coronary intervention, several studies reported an increase of CK-MB and troponin $\mathrm{T}$, suggesting myocardial injury. ${ }^{17-22}$ Furthermore, Regenfus and colleagues ${ }^{23}$ showed that 20 of $48(42 \%)$ patients with successful coronary artery bypass surgery had an increase in LE signal, averaging $5 \%$ of the LV mass. Selvanayagam and colleagues ${ }^{24}$ found a new LE signal in about $40 \%$ of patients undergoing bypass graft surgery. In contrast to these authors, we detected no significant changes in the amount of LE signal in our investigation, suggesting absence of myocardial injury during or after revascularisation in this patient cohort. However, since we do not have information on serum markers (CK$\mathrm{MB}$, troponin) for these patients, we have no proof that no further myocardial injury had occurred.

Of 213 dysfunctional segments with no LE signal at MRI 1, $94(44 \%)$ had improved after revascularisation at MRI 2. This is a strikingly lower percentage than that reported from the studies by Kim et al, ${ }^{11}$ Beek et $a l_{,}^{25}$ and Choi et al, ${ }^{13}$ where almost $80 \%$ had improved function at follow up. A possible explanation for this discrepancy is that the mean time between revascularisation and the follow up scan was around three months in theses studies, whereas it was 8 (4) months (range 3-14 months) in our study. These patients with severe multiple vessel CAD may have had new worsening in function during this longer time period. Moreover, all three mentioned studies included at least a few patients with acute myocardial infarction.

A favourable aspect of the LE signal in contrast enhanced MRI is a high image contrast. Simonetti et $a l^{26}$ and Wu et a ${ }^{27}$ described a signal increase in acute and chronic infarcted myocardium of $485 \%$ and $672 \%$, respectively, compared with remote myocardium after the administration of Gd-DTPA. This high degree of contrast enables accurate differentiation between normal and contrast enhanced myocardium and thus results in low intraobserver and interobserver variabilities as was found in our study. Quantification of the LE signal, which is desirable for an accurate assessment of infarct size in clinical research, may therefore be possible by use of an automatic computer aided threshold analysis, thus providing an objective parameter. An advantage of contrast enhanced MRI over other imaging modalities is high spatial resolution allowing assessment of LE signal transmurality and therefore the ability to identify even small subendocardial infarctions that may otherwise be missed. ${ }^{27}$ Exact assessment of transmurality of the LE signal seems useful, since our and previous investigation ${ }^{11-13}$ have documented a close correlation between the transmural extent of contrast enhancement and the probability of regional functional recovery after revascularisation.

There are some limitations of the study. Firstly, the study was performed with two different MRI scanners and two different imaging sequences. However, every patient was scanned twice with the same scanner and the same imaging sequence by the same physician, guaranteeing the best comparability possible. Moreover, we compared both imaging sequences and found no significant difference in the results between sequences and between MRIs. The best inversion time regarding nulling of the signal in remote myocardium was visually assessed. Newer inversion time scout sequences make this unnecessary and are therefore more reliable. For visual analysis of the LE signal we used a three point scoring system in this study, which had already been published by Klein and colleagues. ${ }^{9}$ In contrast, many other authors divided the transmurality of the LE signal into five categories $(0 \%, 0-25 \%, 25-50 \%, 51-75 \%$, and $>76 \%),{ }^{11} 252728$ which allows a more subtle evaluation of potential changes. Even though MRI provides excellent spatial resolution, we think that an exact classification into five categories is not always that clear cut in this particular patient population with chronic myocardial infarction, where the myocardium appears often very thin. An alternative to visual analysis would be measurement of signal intensities, making the assessment observer independent. However, visual assessment varied very little between and within observers and therefore was reliable for routine use. As mentioned above, some studies reported an increase in LE signal after revascularisation, which we did not find in our patient group B. Since we did not have serum markers (CK-MB, troponin) or complementary nuclear medicine studies available for these patients, we have no proof that no further myocardial injury had occurred.

In conclusion, the results of this study show that the LE signal in patients with chronic CAD and LV impairment is highly biologically and methodologically reproducible over a prolonged time period. This further helps to characterise 
contrast enhanced MRI as a useful tool for myocardial viability assessment. Low intraobserver and interobserver variabilities prove that this method is sufficiently robust for clinical application. Quantitative LE signal assessment may provide an accurate measurement of infarct size as an end point for studies evaluating new treatments.

We thank all technicians in the MRI unit for their excellent support acquiring the studies.

\section{Authors' affiliations}

H Bülow, C Klein, I Kuehn, S G Nekolla, K Schreiber, M Schwaiger, Nuklearmedizinische Klinik der Technischen Universität München, Munich, Germany

R Hollweck, Institut für Medizinische Statistik und Epidemiologie der Technischen Universität München, Munich, Germany

F Haas, J Böhm, R Lange, Abteilung für Herzchirurgie des Deutschen Herzzentrums München, Munich, Germany

B Schnackenburg, Philips Medical Systems, Hamburg, Germany

\section{REFERENCES}

1 Judd RM, Lugo-Olivieri CH, Arai M, et al. Physiological basis of myocardial contrast enhancement in fast magnetic resonance images of 2-day-old reperfused canine infarcts. Circulation 1995;92:1902-10.

2 Kim RJ, Chen EL, Lima JA, et al. Myocardial Gd-DTPA kinetics determine MRI contrast enhancement and reflect the extent and severity of myocardial injury after acute reperfused infarction. Circulation 1996:94:3318-26.

$3 \mathrm{Kim}$ RJ, Fieno DS, Parrish TB, et al. Relationship of MRI delayed contrast enhancement to irreversible injury, infarct age, and contractile function. Circulation 1999;100:1992-2002.

4 Pereira RS, Prato FS, Lekx KS, et al. Contrast-enhanced MRI for the assessment of myocardial viability after permanent coronary artery occlusion. Magn Reson Med 2000:44:309-16.

5 Lima JA, Judd RM, Bazille A, et al. Regional heterogeneity of human myocardial infarcts demonstrated by contrast-enhanced MRI. Potential mechanisms. Circulation 1995:92:1117-25.

6 Laverma K, Niemi P, Hanninen H, et al. Multimodality MR imaging assessment of myocardial viability: combination of first-pass and late contrast enhancement to wall motion dynamics and comparison with FDG PET-initial experience. Radiology 2000;217:729-36.

7 Ramani K, Judd RM, Holly TA, et al. Contrast magnetic resonance imaging in the assessment of myocardial viability in patients with stable coronary artery disease and left ventricular dysfunction. Circulation 1998;98:2687-94.

8 Wagner A, Mahrholdt H, Sechtem U, et al. MR imaging of myocardial perfusion and viability. Magn Reson Imaging Clin N Am 2003;1 1:49-66.

9 Klein C, Nekolla SG, Bengel FM, et al. Assessment of myocardial viability with contrast-enhanced magnetic resonance imaging: comparison with positron emission tomography. Circulation 2002;105:162-7.

10 Mahrholdt H, Wagner A, Holly TA, et al. Reproducibility of chronic infarct size measurement by contrast-enhanced magnetic resonance imaging. Circulation 2002;106:2322-7.

11 Kim RJ, Wu E, Rafael A, et al. The use of contrast-enhanced magnetic resonance imaging to identify reversible myocardial dysfunction. N Engl J Med 2000;343:1445-53
12 Hillenbrand HB, Kim RJ, Parker MA, et al. Early assessment of myocardial salvage by contrast-enhanced magnetic resonance imaging. Circulation 2000;102:1678-83.

13 Choi KM, Kim RJ, Gubernikoff G, et al. Transmural extent of acute myocardial infarction predicts long-term improvement in contractile function. Circulation 2001;104:1101-7.

14 Gerber BL, Garot J, Bluemke DA, et al. Accuracy of contrast-enhanced magnetic resonance imaging in predicting improvement of regional myocardial function in patients after acute myocardial infarction. Circulation 2002;106:1083-9.

15 Reimer KA, Jennings RB. The changing anatomic reference base of evolving myocardial infarction. Underestimation of myocardial collateral blood flow and overestimation of experimental anatomic infarct size due to tissue edema, hemorrhage and acute inflammation. Circulation 1979;60:866-76

16 van Dijkman PR, Hold KM, van der Laarse A, et al. Sequential analysis of infarcted and normal myocardium in piglets using in vivo gadoliniumenhanced MR images. Magn Reson Imaging 1993;11:207-18.

17 Costa MA, Carere RG, Lichtenstein SV, et al. Incidence, predictors, and significance of abnormal cardiac enzyme rise in patients treated with bypass surgery in the arterial revascularization therapies study (ARTS). Circulation $2001 ; 104: 2689-93$

18 Januzzi JL, Lewandrowski K, MacGillivray TE, et al. A comparison of cardiac troponin $T$ and creatine kinase-MB for patient evaluation after cardiac surgery. J Am Coll Cardiol 2002;39:1518-23.

19 Dahlin LG, Kagedal B, Nylander E, et al. Early identification of permanent myocardial damage after coronary surgery is aided by repeated measurements of CK-MB. Scand Cardiovasc J 2002;36:35-40.

20 Brener SJ, Lytle BW, Schneider JP, et al. Association between CK-MB elevation after percutaneous or surgical revascularization and three-year mortality. J Am Coll Cardiol 2002;40:1961-7.

21 Klatte K, Chaitman BR, Theroux P, et al. Increased mortality after coronary artery bypass graft surgery is associated with increased levels of postoperative creatine kinase-myocardial band isoenzyme release: results from the GUARDIAN trial. J Am Coll Cardiol 2001;38:1070-7.

22 Shiga T, Terajima K, Matsumura J, et al. Minor cardiac troponin T release in patients undergoing coronary artery bypass graft surgery on a beating heart. $J$ Cardiothorac Vasc Anesth 2000;14:151-5.

23 Regenfus $M$, Wagner $A, H$ M, et al. Impact of myocardial infarction associated with successful coronary artery bypass surgery on post-operative contractile improvement. Circulation 2002;106(suppl II):390.

24 Selvanayagam JB, Petersen SE, Francis JM, et al. Effects of off-pump versus on-pump coronary surgery on reversible and irreversible myocardial injury: a randomized trial using cardiovascular magnetic resonance imaging and biochemical markers. Circulation 2004;109:345-50.

25 Beek AM, Kuhl HP, Bondarenko O, et al. Delayed contrast-enhanced magnetic resonance imaging for the prediction of regional functiona improvement after acute myocardial infarction. J Am Coll Cardiol 2003;42:895-901.

26 Simonetti OP, Kim RJ, Fieno DS, et al. An improved MR imaging technique for the visualization of myocardial infarction. Radiology 2001;218:215-23.

27 Wu E, Judd RM, Vargas JD, et al. Visualisation of presence, location, and transmural extent of healed $Q$-wave and non-Q-wave myocardial infarction. Lancet $2001 ; 357: 21-8$.

28 Wagner A, Mahrholdt H, Holly TA, et al. Contrast-enhanced MRI and routine single photon emission computed tomography (SPECT) perfusion imaging for detection of subendocardial myocardial infarcts: an imaging study. Lancet 2003;361:374-9

\section{Call for papers}

11 th European Forum on Quality Improvement in Health Care

26-28 April 2006, Prague, Czech Republic

Deadline 30 September 2005.

For further information and to submit online go to: www.quality.bmipg.com 